\title{
STEPHAN TIGAN
}

\section{M. STANCU-MINASIAN}

\section{Methods for solving stochastic bilinear fractional max-min problems}

Revue française d'automatique, d'informatique et de recherche opérationnelle. Recherche opérationnelle, tome 30, n 1 (1996), p. 81-98.

<http://www.numdam.org/item?id=RO_1996_30_1_81_0>

(C) AFCET, 1996, tous droits réservés.

L'accès aux archives de la revue «Revue française d'automatique, d'informatique et de recherche opérationnelle. Recherche opérationnelle » implique l'accord avec les conditions générales d'utilisation (http://www.numdam.org/ legal.php). Toute utilisation commerciale ou impression systématique est constitutive d'une infraction pénale. Toute copie ou impression de ce fichier doit contenir la présente mention de copyright.

\section{Numdam}




\title{
METHODS FOR SOLVING STOCHASTIC BILINEAR FRACTIONAL MAX-MIN PROBLEMS (*)
}

\author{
by Stephan Tigan $\left({ }^{1}\right)$ and I. M. Stancu-Minasian $\left({ }^{2}\right)$ \\ Communicated by Jean-Yves JAFFRAY
}

\begin{abstract}
In this paper we consider a stochastic bilinear fractional max-min problem with separate linear constraints. In the case when all the coefficients of the objective function are simple randomized, two ways for solving this problem will be employed: the minimum-risk approach and Kataoka's model.

We prove that, under some positivity conditions, these stochastic problems are equivalent with certain deterministic bilinear-fractional min-max (or max-min) problems. For solving these deterministic optimization problems a parametrical procedure will be presented.

Keywords: Stochastic programming, Bilinear fractional programming, Minimum risk approach, Min-max problems.

Résumé. - Dans cet article nous considérons une classe de problèmes stochastiques bilinéairesfractionnaires avec contraintes linéaires séparées. Si les coefficients de la fonction objective sont simplement randomisés nous présentons deux voies pour la résolution de ces problèmes: la méthode du risque minimal et le modèle de Kataoka. Nous démontrons, sous certaines conditions de positivité sur la fonction objective, que ces problèmes stochastiques sont équivalents avec certains problèmes déterministes de min-max (ou max-min) avec une fonction objective bilinéaire-fractionnaire. Pour ces problèmes déterministes nous proposons une procédure paramétrique de résolution.
\end{abstract}

Mots clés : Programmation stochastique, Programmation bilinéaire-fractionnaire, Problème du risque minimal, Problèmes de min-max.

\section{INTRODUCTION}

In this paper, a stochastic bilinear fractional max-min problem with separate linear constraints is considered. In the case when all the coefficients of the objective function are simple randomized, two ways for solving this problem will be employed: the minimum-risk approach (see, e.g. [2, 15, 16, 19, 20]) and Kataoka's model [11].

(*) Received April 1994.

(') Univ. of Medicine and Pharmacy Cluj-Napoca Str. Pasteur No.6, 3400 Cluj-Napoca, Romania.

$\left({ }^{2}\right)$ Centre of Mathematical Statistics, the Romanian Academy, Bd. Magheru 22, RO-70158 Bucharest 22, Romania.

Recherche opérationnelle/Operations Research, 0399-0559/96/01/\$ 4.00

(c) AFCET-Gauthier-Villars 
We show that, under some positivity conditions, these stochastic problems are equivalent with certain deterministic bilinear-fractional min-max (or maxmin) problems, for which a parametrical procedure will be presented. Some remarks concerning the convergence of this procedure will be made.

Likewise assuming that only the denominator of the objective function is random, a particular case will be studied by combining the variable transformation of Charnes-Cooper type [4] with the minimum-risk approach.

We consider also the case when only part of the coefficients of the objective function are random with a normal distribution. In this case, for the minimumrisk problem and Kataoka's model we obtain some deterministic equivalent fractional max-min problems.

\section{PROBLEM FORMULATION}

Consider the following bilinear-fractional max-min problem:

P. Find

$$
\max _{x \in X} \min _{y \in Y} \frac{x A y+a x+b y+c}{x B y+d x+e y+f}
$$

where

$$
\begin{aligned}
& X=\left\{x \in R^{n}: C x \leq g, x \geq 0\right\}, \\
& Y=\left\{y \in R^{m}: D y \leq h, y \geq 0\right\},
\end{aligned}
$$

and $x$ and $y$ are variable vectors, whereas $A, B \in R^{n \times m}, C \in R^{p \times n}$, $D \in R^{q \times m}, a, d \in R^{n}, b, e \in R^{m}, g \in R^{p}, h \in R^{q}, c, f \in R$ are given matrices, vectors and real numbers respectively.

As can be seen, the constraints in problem (2.1)-(2.3) are separate. Such max-min programs have applications, for instance, in the fields of polyhedral games (Wolfe [31], Schroeder [13]), of multiobjective programming (Soyster [14], Tigan and Stancu-Minasian [22], [30]), and of scheduling problems (Belenkii [1]).

Other extensions of problems $P$ refer to pseudofractional max-min programming [26] and generalized fractional max-min problems [21] with generalized activity coefficients in Dantzig's sense [7].

We make the remark that certain max-min problems with linked linear constraints and quasimonotonic objective functions were studied in [6], [25], [28] and some stochastic max-min problems with linked linear constraints and linear or linear-fractional objectives were considered in [20] and [29]. 
An example of the bilinear-fractional max-min problem (P) which has a theoretical importance is given by Golstein [10]. Golstein proves that the following generalized fractional problem:

GFP. Find

$$
\max _{x \in X} \min _{1 \leq i \leq m} \frac{f_{i}(x)}{g_{i}(x)}
$$

can be reduced to a bilinear max-min problem with separate linear constraints. In the formulation of problem GFP, the set $X$ is defined by (2.2) and $f_{i}$ and $g_{i}$ are affine functions of the form:

$$
f_{i}(x)=x a^{i}+a^{0 i}, \quad g_{i}(x)=x b^{i}+b^{0 i}, \quad i=1,2, \ldots, m,
$$

where $a^{i}$ and $b^{i}$ are given column vectors in $R^{n}$ and $a^{0 i}$ and $b^{0 i}$ are given real numbers. Under the assumption of strict positivity of functions $g_{i}$ on the feasible set $X$, Golstein [10] shows that problem GFP is equivalent with the following bilinear max-min problem with separate feasible sets:

MMP. Find

$$
\max _{x \in X} \min _{y \in Y} \frac{f_{1}(x) y_{1}+\ldots+f_{m}(x) y_{m}}{g_{1}(x) y_{1}+\ldots+g_{m}(x) y_{m}}
$$

where

$$
Y=\left\{y \in R^{m} / y_{1}+\ldots+y_{m}=1, y \geq 0\right\} .
$$

But the problem MMP is a particular case of the bilinear max-min problem $P$.

Next we adopt the following usual assumptions for fractional max-min problem $P$ :

$$
x B y+d x+e y+f>0, \quad \text { for all }(x, y) \in X \times Y .
$$

Definition 1: Given a function $F: S \times Q \rightarrow R$, ( $S$ subset of $R^{n^{\prime}}$ and $Q$ subset of $\left.R^{m \prime}\right)$, we say that $\left(x^{\prime}, y^{\prime}\right) \in S \times Q$ is an optimal solution for the max-min problem:

$$
\max _{x \in S} \min _{y \in Q} F(x, y)
$$


if $\left(x^{\prime}, y^{\prime}\right)$ verifies the following conditions:

$$
\begin{gathered}
F\left(x^{\prime}, y^{\prime}\right)=\min \left\{F\left(x^{\prime}, y\right): y \in Q\right\} \\
F\left(x^{\prime}, y^{\prime}\right) \geq \min \{F(x, y): y \in Q\}, \quad \text { for all } x \in S .
\end{gathered}
$$

In [17], we proposed a parametrical method for solving problem $P$ (see also [24], [27]).

\section{THE MINIMUM-RISK APPROACH. THE SIMPLE RANDOMIZATION CASE}

In what follows, we shall assume that the matrices $A, B$ and the vectors $a, b, d, e$ are random with simple randomization, i.e. of the form:

$$
\begin{array}{cc}
A(w)=A^{\prime}+t(w) A^{\prime \prime}, & B(w)=B^{\prime}+t(w) B^{\prime \prime} \\
a(w)=a^{\prime}+t(w) a^{\prime \prime}, & b(w)=b^{\prime}+t(w) b^{\prime \prime} \\
d(w)=d^{\prime}+t(w) d^{\prime \prime}, & e(w)=e^{\prime}+t(w) e^{\prime \prime}
\end{array}
$$

where $A^{\prime}, A^{\prime \prime}, B^{\prime}, B^{\prime \prime} \in R^{n \times m}$ are given matrices, $a^{\prime}, a^{\prime \prime}, d^{\prime}, d^{\prime \prime} \in R^{n}$, $b^{\prime}, b^{\prime \prime}, e^{\prime}, e^{\prime \prime} \in R^{m}$ are given constant vectors, whereas $t(w)$ is a random variable on a probability space $(\Omega, K, P)$ with a continuous and strictly increasing distribution function $T$.

The minimum-risk problem corresponding to level $z$, associated to problem $P$, consists in finding the optimal solution of the following problem:

PR. Find

$$
v(z)=\max _{x \in X} P\{w: Z(x, w)>z\}
$$

where

$$
Z(x, w)=\min \{H(x, y, w): y \in Y\}
$$

and

$$
H(x, y, m)=\frac{x A(w) y+a(w) x+b(w) y+c}{x B(w) y+d(w) x+e(w) y+f}
$$


Concerning problem PR, we make an assumption similar to $\mathrm{H} 1$ :

$$
\left.\begin{array}{c}
x B(w) y+d(w) x+e(w) y+f>0 \\
\forall(x, y) \in X \times Y \quad \text { and } \quad \forall w \in \Omega .
\end{array}\right\}
$$

Definition 2: A pair $\left(x^{\prime}, y^{\prime}\right) \in X \times Y$ is called minimum-risk solution for problem PR, if the following conditions hold:

$$
\begin{gathered}
P\left\{w: H\left(x^{\prime}, y^{\prime}, w\right)>z\right\} \\
=P\left\{w: \min \left\{H\left(x^{\prime}, y, w\right): y \in Y\right\}>z\right\}, \\
P\left\{w: H\left(x^{\prime}, y^{\prime}, w\right)>z\right\} \\
\geq P\{w: \min \{H(x, y, w): y \in Y\}>z\}, \text { for all } x \in X .
\end{gathered}
$$

We note that the minimum-risk approach was introduced in stochastic linear programming by Bereanu $[2,3]$ and Charnes and Cooper [5] (the P-model). This approach is extended by Stancu-Minasian [15] to the stochastic programming with linear-fractional objective and a product of two linear functions and by Stancu-Minasian and Tigan $[16,19]$ to Tchebyshev linear-fractional stochastic problems. Denote:

$$
U(x, y)=x\left(z B^{\prime}-A^{\prime}\right) y+\left(z d^{\prime}-a^{\prime}\right) x+\left(z e^{\prime}-b^{\prime}\right) y+z f-c
$$

and

$$
\begin{gathered}
W(x, y)=x\left(A^{\prime \prime}-z B^{\prime \prime}\right) y+\left(a^{\prime \prime}-z d^{\prime \prime}\right) x+\left(b^{\prime \prime}-z e^{\prime \prime}\right) y \\
\text { for all }(x, y) \in X \times Y
\end{gathered}
$$

Further, we shall suppose that:

$$
W(x, y)>0, \quad \text { for all }(x, y) \in X \times Y
$$

$X$ and $Y$ are bounded and nonempty sets. 
The next theorem shows how under the assumptions (3.8) and (3.9), the minimum-risk problem PR, can be solved by a deterministic problem, which does not depend on the distribution function of the random variable $t(w)$. This property generalises a similar result obtained in [17] for bilinear programming.

THEOREM 1: If conditions (3.8) and (3.9) hold and if the distribution function $T$ is continuous and strictly increasing, then the minimum-risk solution of problem $P R$ does not depend on $T$ and it can be obtained by solving the following min-max bilinear problem:

$$
\text { PA. } \min _{x \in X} \max _{y \in Y} \frac{U(x, y)}{W(x, y)} .
$$

Proof: From (3.1)-(3.5), we get:

$$
\begin{aligned}
G(x, z) & =P\{w: \min \{H(x, y, w): y \in Y\}>z\} \\
& =P\{w: H(x, y, w)>z, \text { for all } y \in Y\} .
\end{aligned}
$$

Hence, according to (3.8), it results that

$$
\begin{aligned}
G(x, z) & =P\{w: t(w)>U(x, y) / W(x, y), \text { for all } y \in Y\} \\
& =P\{w: t(w)>\max \{U(x, y) / W(x, y): y \in Y\}\} \\
& =1-P\{w: t(w)<\max \{U(x, y) / W(x, y): y \in Y\}\}
\end{aligned}
$$

Then the PR problem is equivalent to:

$$
\begin{aligned}
& \max \{G(x, w): x \in X\} \\
& \quad=\max \{1-T(\max \{U(x, y) / W(x, y): y \in Y\}): x \in X\}
\end{aligned}
$$

Therefore, by the assumption that $T$ is continuous and strictly increasing, we get:

$$
v(z)=\max \{G(x, z): x \in X\}=1-T\left(\min _{x \in X} \max _{y \in Y} \frac{U(x, y)}{W(x, y)}\right)
$$

which implies that a minimum-risk solution for problem PR can be obtained by solving problem PA. 


\section{KATAOKA'S MODEL. THE SIMPLE RANDOMIZATION CASE}

Related to minimum-risk problem $\mathrm{PR}$ is the following generalized Kataoka's problem [11], which can be associated to problem $P$ :

PK. Find

\section{$\max z$}

subject to

$$
\begin{gathered}
P\left\{w: \min _{y \in Y} H(x, y ; w) \geq z\right\} \geq \alpha, \\
x \in X, \quad z \in R,
\end{gathered}
$$

where $\alpha \in[0,1]$ is a given lower level for the probability in (4.1).

Next, let assume that in the fractional objective function $H$ (see (3.5)) only the numerator is random, that is, in (3.1), (3.2) and (3.3), one has:

$$
B^{\prime \prime}=0, \quad d^{\prime \prime}=0, \quad e^{\prime \prime}=0, \quad B=B^{\prime}, \quad d=d^{\prime}, \quad e=e^{\prime} .
$$

Moreover, we suppose that

$$
D^{\prime \prime}(x, y)=x A^{\prime \prime} y+a^{\prime \prime} x+b^{\prime \prime} y>0, \quad \forall(x, y) \in X \times Y,
$$

and, for any $(x, y) \in X \times Y$, we denote

$$
\begin{gathered}
D^{\prime}(x, y)=x A^{\prime} y+a^{\prime} x+b^{\prime} y+c, \\
N(x, y)=x B y+d x+e y+f .
\end{gathered}
$$

THEOREM 2: If assumptions H1, (3.9), (4.3) and (4.4) hold and if the distribution function $T$ is continuous and strictly increasing, then any optimal solution of problem PK can be obtained by solving the following bilinear fractional max-min problem:

PKA. Find

$$
\max _{x \in X} \min _{y \in Y} \frac{D^{\prime}(x, y)+T^{-1}(1-\alpha) D^{\prime \prime}(x, y)}{N(x, y)} .
$$


Proof: Indeed, by $\mathrm{H} 1$ and (4.4), we have

$$
\begin{aligned}
P & \left\{w: \min _{y \in Y} H(x, y ; w) \geq z\right\}=P\{w: H(x, y, w) \geq z, \forall y \in Y\} \\
& =P\{w: x A(w) y+a(w) x+b(w) y+c \geq z N(x, y), \forall y \in Y\} \\
& =P\left\{w: t(w) \geq \frac{z N(x, y)-D^{\prime}(x, y)}{D^{\prime \prime}(x, y)}, \forall y \in Y\right\} \\
& =P\left\{w: t(w) \geq \max _{y \in Y} \frac{z N(x, y)-D^{\prime}(x, y)}{D^{\prime \prime}(x, y)}\right\} \\
& =1-T\left\{\max _{y \in Y} \frac{z N(x, y)-D^{\prime}(x, y)}{D^{\prime \prime}(x, y)}\right\} .
\end{aligned}
$$

But, then the inequality (4.1), may be rewritten as:

$$
\max _{y \in Y} \frac{z N(x, y)-D^{\prime}(x, y)}{D^{\prime \prime}(x, y)} \leq T^{-1}(1-\alpha),
$$

which by $\mathrm{H} 1$ and (4.4) is equivalent to

$$
z \leq \min _{y \in Y} \frac{D^{\prime}(x, y)+T^{-1}(1-\alpha) D^{\prime \prime}(x, y)}{N(x, y)} .
$$

The last inequality implies obviously that any optimal solution of problem PK may be obtained by solving max-min bilinear fractional problem PKA.

\section{A PARTicular CASE}

In this section, we consider a particular case of problem PR, when only the objective denominator is random, while the objective nominator has a deterministic special form. In this max-min problem, denoted by PRF, the objective function $H$ is expressed as

$$
H(x, y, w)=\frac{x A(w) y+a(w) x+b(w) y+c}{(d x+f)(e y+r)}
$$

for all $(x, y) \in X \times Y$ and $w \in \Omega$.

In (5.1), $d \in R^{n}, e \in R^{m}, f, r \in R$ are given real vectors and numbers respectively, and the other notations have the same meaning as in the statement of problem PR. 
From problem PRF, performing the variable transformations (see $[4,12,23])$

$$
u=\theta x, \quad v=s y
$$

we get the following minimum risk problem

PRL. Find

$$
V^{\prime}(z)=\max _{(u, \Theta) \in X^{\prime}} P\left\{w: Z^{\prime}(u, \theta, w)>z\right\}
$$

where:

$$
\begin{gathered}
X^{\prime}=\left\{(u, \theta) \in R^{n+1}: C u-g \theta \leq 0, d u+f \theta=1, u, \theta \geq 0\right\} \\
Z^{\prime}(u, \Theta, w)=\min \left\{H^{\prime}(u, \theta, v, s, w):(v, s) \in Y^{\prime}\right\} \\
H^{\prime}(u, \theta, v, s, w)=u A(w) v+a(w) s u+b(w) \theta v+c \theta s \\
Y^{\prime}=\left\{(v, s) \in R^{m+1}: D v-h s \leq 0, e v+r s=1, v, s \geq 0\right\}
\end{gathered}
$$

Suppose that:

$$
\begin{aligned}
& d x+d>0, \quad \text { for all } x \in X \\
& e y+r>0, \quad \text { for all } y \in Y
\end{aligned}
$$

THEOREM 3: Assume that (3.9), (5.8) and (5.9) hold. Then we have:

(i) if $(u, \theta) \in X^{\prime}$ and $(v, s) \in Y^{\prime}$, then $\theta$ and $s$ are strictly positive numbers;

(ii) if $\left(u^{\prime}, \theta^{\prime}, v^{\prime}, s^{\prime}\right)$ is a minimum-risk solution for $P R L$, then $\left(u^{\prime} / \theta^{\prime}, v^{\prime}\right) / s^{\prime}$ is a minimum-risk solution for PRF.

Proof: Assertion (i) can be easily proven by using assumption (3.9) (see, e.g., Charnes-Cooper [4]). Part (ii) of the theorem follows by performing the variable transformation (5.2) in problem PRF. 
Theorem 3 provides an approach to transform the fractional max-min problem PRF into a bilinear stochastic max-min problem [17] (problem PRL), which, by Theorem 1 (see, also [17]), is equivalent with a deterministic bilinear-fractional max-min problem.

\section{THE CASE OF NORMAL DISTRIBUTION}

In this section we suppose that in problem $P$ (see (2.1)-(2.3)), only the vector $a$ is an $n$-dimensionl random vector defined on a probability space $(\Omega, K, P)$.

We adopt particularly the following assumptions:

H3) The costs $a_{i}(i \in\{1,2, \ldots, n\})$ are normal random variables.

H4) The feasible set $X$ does not contain zero vector.

If the vector $a$ has the mean value $a^{*}$ and the covariance matrix $V$, then, under the assumption $\mathrm{H} 3, a x$ is a normal random variable of type $N\left(a^{*} x ; x V x\right)$.

In this case, the minimum-risk problem corresponding to level $z$, associated to problem (2.1)-(2.3), consists in finding the optimal solution of the following problem:

PN. Find

$$
v^{\prime \prime}(z)=\max _{x \in X} P\left\{w: \min _{y \in Y} \frac{x A y+a(w) x+b y+c}{x B y+d x+e y+f} \geq z\right\} .
$$

Denote

$$
\left.\begin{array}{c}
H(x, y ; w)=\frac{x A y+a(w) x+b y+c}{x B y+d x+e y+f}, \\
\text { for any } x \in X, y \in Y \text { and } w \in \Omega
\end{array}\right\}
$$

The minimum-risk solution of problem PN is defined as for problem PR (see, Definition 2).

THEOREM 4: If assumptions $\mathrm{HI}, \mathrm{H} 3$ and $\mathrm{H} 4$ hold, then any minimum-risk solution of problem PN is given by the solution of the following fractional max-min problem:

$P N F$. Find

$$
\max _{x \in X} \min _{y \in Y} \frac{\left(a^{*}-z d\right) x+x(A-z B) y+(b-z e) y+c-z f}{(x V x)^{\frac{1}{2}}}
$$


Proof: Indeed, by assumption $\mathrm{H} 1$, for any $x \in X$, we have:

$$
\begin{aligned}
& Z(x ; z) \\
& \quad=P\left\{w: \min _{y \in Y} H(x, y, w) \geq z\right\} \\
& =P\{w: H(x, y, w) \geq z, \forall y \in Y\} \\
& =P\{w: a(w) x \geq z d x+x(z B-A) y \\
& \quad+(z e-b) y+z f-c, \forall y \in Y\} .
\end{aligned}
$$

But, since $a(w) x$ is a normal random variable of the type $N\left(a^{*} x ; x V x\right)$, we get:

$$
\begin{aligned}
& Z(x ; z) \\
& =P\left\{w: \frac{a(w) x-a^{*} x}{(x V x)^{\frac{1}{2}}} \geq \frac{\left\{\begin{array}{c}
z d x+x(z B-A) y \\
+(z e-b) y+z f-c-a^{*} x
\end{array}\right\}}{\forall y \in Y(x V x)^{\frac{1}{2}}},\right\} \\
& =P\left\{w: \frac{a(w) x-a^{*} x}{(x V x)^{\frac{1}{2}}} \geq \max _{y \in Y} \frac{\left\{\begin{array}{c}
z d x+x(z B-A) y \\
+(z e-b) y+z f-c-a^{*} x
\end{array}\right\}}{(x V x)^{\frac{1}{2}}}\right\} \\
& =1-\Phi\left\{\max _{y \in Y} \frac{z d x+x(z B-A) y+(z e-b) y+z f-c-a^{*} x}{(x V x)^{\frac{1}{2}}}\right\},
\end{aligned}
$$

where

$$
\Phi(t)=\frac{1}{(2 \Pi)^{\frac{1}{2}}} \int_{-\infty}^{t} \exp \left(-s^{2} / 2\right) d s
$$

is the Laplace function.

vol. $30, \mathrm{n}^{\circ} 1,1996$ 
Since $\Phi$ is a non-decreasing and continuous function, it results that

$$
\begin{aligned}
v^{\prime \prime}(z) & =\max _{x \in X} Z(x ; z) \\
& =1-\Phi\left\{\max _{x \in X} \min _{y \in Y} \frac{\left\{\begin{array}{c}
z d x+x(z B-A) y \\
+(z e-b) y+z f-c-a^{*} x
\end{array}\right\}}{(x V x)^{\frac{1}{2}}}\right\} .
\end{aligned}
$$

But (6.3) implies that $\mathrm{PN}$ is equivalent with fractional max-min problem PNF.

Related to the minimum-risk problem PN, we can consider the following Kataoka's problem:

PKN. Maximize $z$ subject to

$$
P\{w: \min H(x, y, w) \geq z\} \geq \alpha, \quad x \in X, \quad z \in R,
$$

where $H$ is defined by (6.2) and $\alpha$ is a given number in the interval $[0,1]$.

THEOREM 5: If assumption $\mathrm{HI}, \mathrm{H} 3$ and $\mathrm{H} 4$ hold, then any optimal solution of problem PKN is given by the solution of the following fractional max-min program:

KNA. Find

$$
\max _{x \in X} \min _{y \in Y} \frac{D^{1}(x, y)+\Phi^{-1}(1-\alpha)(x V x)^{\frac{1}{2}}}{N(x, y)}
$$

where

$$
D^{1}(x, y)=x A y+a^{*} x+b y+c .
$$

Proof: Following the same reasoning as in the proof of theorem 4, we have

$$
\begin{aligned}
P & \left\{w: \min _{y \in Y} H(x, y, w) \geq z\right\} \\
& =1-\Phi\left\{\max _{y \in Y} \frac{z N(x, y)-D^{1}(x, y)}{(x V x)^{\frac{1}{2}}}\right\} .
\end{aligned}
$$


But, then (6.4), is equivalent to

$$
\max _{y \in Y} \frac{z N(x, y)-D^{1}(x, y)}{(x V x)^{\frac{1}{2}}} \leq \Phi^{-1}(1-\alpha),
$$

which, by the assumptions $\mathrm{H} 1$ and $\mathrm{H} 4$, is equivalent to

$$
z \leq \min _{y \in Y} \frac{D^{1}(x, y)+\Phi^{-1}(1-\alpha)(x V x)^{\frac{1}{2}}}{N(x, y)} .
$$

But the last inequality implies that any optimal solution of problem PKN may be obtained by solving fractional max-min problem PKNA.

The fractional max-min problems PNF and PKNA, under some additional hypotheses, may be solved by the pseudo-quadratic programming technique developed in $[8,9]$.

\section{THE ITERATIVE NUMERICAL PROCEDURE}

In this section, we will present a numerical method for solving the deterministic bilinear fractional min-max problem PA, and, by Theorems 1 and 2, the stochastic problems PR (or PRF). A similar method can be used for solving the bilinear fractional max-min problem PKA.

With this aim in view, we employ a parametrical procedure which represents a specific particularization to problem PA of a general iterative method given in [24] and [27] (see, also [17, 18]).

The following theorem gives a necessary and sufficient condition for a pair $\left(x^{\prime}, y^{\prime}\right) \in X \times y$, to be an optimal solution for min-max problem PM.

THEOREM 6: Let $x^{\prime} \in X$ and $t^{\prime} \in R$ be such that:

$$
t^{\prime}=\frac{U\left(x^{\prime}, y^{\prime}\right)}{W\left(x^{\prime}, y^{\prime}\right)}=\max _{y \in Y} \frac{U\left(x^{\prime}, y\right)}{W\left(x^{\prime}, y\right)} .
$$

Then $\left(x^{\prime}, y^{\prime}\right)$ is an optimal solution for PA if and only if $\beta\left(t^{\prime}\right)=0$, where for any $t$ in $R$ :

$$
\beta(t)=\min _{x \in X} \max _{y \in Y}(U(x, y)-t W(x, y)) .
$$

Proof: The proof is similar to that of Theorem 1 from ref. [17].

The parametrical procedure for solving problem PA consists in the following steps. 
Parametrical algorithm

Step 1. Choose $x_{0} \in X$ and take $k=0$.

Step 2. Solve the linear-fractional program:

$$
t_{k}=\max _{y \in Y} \frac{U\left(x_{k}, y\right)}{W\left(x_{k}, y\right)}
$$

and let $y_{k} \in Y$ be an optimal solution for (7.1).

Step 3. Solve the min-max bilinear problem:

$$
\beta\left(t_{k}\right)=\min _{x \in X} \max _{y \in Y}\left(U(x, y)-t_{k} W(x, y)\right),
$$

and let $\left(x_{k+1}, y_{k+1}^{\prime}\right)$ be an optimal solution of this problem.

Step 4. i) If $\beta\left(t_{k}\right)=0$, then by Theorem $6,\left(x_{k}, y_{k}\right)$ is an optimal solution for problem PA, and the procedure stops 2 .

ii) If $\beta\left(t_{k}\right)<0$, take $k:=k+1$ and go to Step 2 .

An optimal solution obtained by the parametrical procedure (for min-max problem PA) is also, by Theorem 1, a minimum-risk solution for problem PR.

The optimal value $\beta\left(t_{k}\right)$ of the min-max problem (7.2) in Step 3, may be obtained by solving a classical linear program (see, e.g. [19]):

$\operatorname{PL}\left(t_{k}\right)$. Find

$$
\beta\left(t_{k}\right)=\min _{x, \mu}\left(M_{k} x+h \mu+z f-c\right)
$$

subject to:

$$
\begin{aligned}
& \mu D \geq x L_{k}+N_{k}, \\
& \mu \geq 0, \quad x \in X,
\end{aligned}
$$

where $\mu \in R^{q}$ is the vector of dual variables and

$$
\begin{aligned}
M_{k} & =\left(z d^{\prime}-a^{\prime}\right)-t_{k}\left(a^{\prime \prime}-z d^{\prime \prime}\right) \\
N_{k} & =\left(z e^{\prime}-b^{\prime}\right)-t_{k}\left(b^{\prime \prime}-z e^{\prime \prime}\right) \\
L_{k} & =\left(z B^{\prime}-A^{\prime}\right)-t_{k}\left(A^{\prime \prime}-z B^{\prime \prime}\right) .
\end{aligned}
$$


We mention that problem $P L\left(t_{k}\right)$ is obtained from problem (7.2), by applying, for every $x \in X$, the linear programming duality to the linear program:

$$
\max _{y}\left\{U(x, y)-t_{k} W(x, y): D y \leq h, y \geq 0\right\}
$$

Then Step 3, can be replaced by the following step: Step $3^{\prime}$. Solve the linear program $P L\left(t_{k}\right)$. Let $\beta\left(t_{k}\right)$ be the optimal value and let $\left(x_{k+1}, \mu_{k+1}\right)$ be an optimal solution of program $P L\left(t_{k}\right)$.

\section{ALGORITHMIC REMARKS}

Since the parametrical procedure generally needs an infinite number of iterations, in order to obtain a finite iterative method, an approximate stop criterion may be used in Step 4:

Step $4^{\prime}$. i) If $\left|\beta\left(t_{k}\right)\right| \leq r^{\prime}$, then the algorithm stops and $\left(x_{k}, y_{k}\right)$ is an approximate solution for problem PA (or problem PR).

ii) If $\left|\beta\left(t_{k}\right)\right|>r^{\prime}$, then go to Step 2 with $k$ replaced by $k+1$.

Here, $r^{\prime}$ is a positive number which represents a measure of the desired approximation.

The algorithm convergence, as well as the finiteness of the approximate variant is based on

THEOREM 7: Let $r^{\prime} \geq 0$ be a given real number. Under hypotheses (3.8) and (3.9), if $-r^{\prime} \leq \beta\left(t_{k}\right) \leq 0$ and if $W(x, y) \geq s^{\prime}>0$, for every $(x, y) \in X \times Y$, then, for every optimal solution of problem $P A$, the following inequalities hold:

$$
0 \leq\left|t_{k}-\frac{U\left(x^{\prime}, y^{\prime}\right)}{W\left(x^{\prime}, y^{\prime}\right)}\right| \leq\left|\beta\left(t_{k}\right)\right| / s^{\prime} \leq r^{\prime} / s^{\prime} .
$$

Proof: The proof of the theorem is similar to that of Theorem 3, from Ref. [24].

This algorithmic approach needs to solve at each iteration, by simplex techniques, only two programming problems: a linear-fractional program in Step 2, and an ordinary linear programming problem in Step 3.

At iteration $k$, the solving of the linear-fractional program (7.1) in Step 2 may begin, except the first iteration, with $y_{k-1}$ as initial solution (the optimal solution of (7.1) obtained in the previous iteration). 
In Step 3, for solving the linear program $P L\left(t_{k}\right)$, a primal-dual simplex algorithm may be suitably used, in order to employ as initial solution, at iteration $k$, the optimal solution obtained in the previous iteration. This approach seems to be especially efficient when the number of constraints which define the set $X$ is great with respect to the number $q$ of constraints (7.3).

\section{CONCLUSIONS}

Two ways for solving stochastic bilinear fractional max-min problems with separate linear constraints are discussed: the minimum-risk approach and Kataoka's model. In the case when the objective function is simple randomized some deterministic equivalent bilinear fractional min-max (or max-min) problems are obtained, and a parametrical procedure involving only linear and linear fractional programming techniques for solving such problems are presented.

When the objective function has certain random coefficients with a normal distribution, we show that, under some regularity conditions, for the corresponding minimum-risk and Kataoka's problems can be also obtained equivalent deterministic fractional max-min problems, which are not generally bilinear (e.g., problems PNF and PKNA). Under some additional assumtions, the pseudo-quadratic programming techniques can be used for solving these problems.

Similar results to the Theorem 1.2 can be obtained for the case when the domains of $x$ and $y$ are nonseparated, but in this case we can not use the proposed algorithm for solving the equivalent deterministic problems.

\section{ACKNOWLEGMENTS}

The authors wish to express their sincere appreciation to prof. Jean-Yves Jaffray for his many comments and suggestions.

\section{REFERENCES}

1. A. S. BeLENKII, Minimax scheduling problems with linear constraints and methods of their solutions, Avt. i Telemeh., 1981, 10, pp. 157-170.

2. B. Bereanu, On stochastic linear programming, I: Distribution problems: A simple random variable, Revue Roumaine Math. Pures Appl., 1963, 8 (4), pp. 683-697.

3. B. Bereanu, Programme de risque minimal en programmation linéaire stochastique, C. R. Acad. Sci. Paris, 1964, 259, pp. 981-983. 
4. A. Charnes and W. W. Cooper, Programming with linear fractional functionals, Naval Res. Logist. Quart., 1962, 9 (3-4), pp. 181-186.

5. A. Charnes and W. W. Cooper, Deterministic equivalents for optimizing and satisfying under change constraints, Operations Res., 1963, 11, pp. 18-39.

6. W. D. Cook, M. J. L. Kirby and S. L. MehndiratTa, A linear fractional max-min problem, Operations Res., 1975, 23 (3), pp. 511-521.

7. G. B. Dantzig, Linear Programming and Extensions, Princeton Univ. Press, Princeton, New-Jersey, 1963.

8. M. Dragomirescu, An algorithm for the minimum-risk problem of stochastic programming, Operations Res., 1972, 30 (1), pp. 154-164.

9. M. Dragomirescu and M. Malita, Programare neliniara, Editura Stiintifica, Bucuresti, 1972.

10. E. G. Golstein, Duality theory in mathematical programming and its applications, Nauka, Moskva, 1971, (in russian).

11. S. KатАокA, A stochastic programming model, Econometrica, 1963, 31, pp. 181-196.

12. S. Schaible, Nonlinear Fractional Programming, Oper. Res. Verfahren, 1974, 19, pp. 109-115.

13. R. G. Schroeder, Linear programming solutions to ratio games, Operations Res., 1970,18 , pp. $300-305$.

14. A. L. Soyster, B. Lev and Di Toof, Conservative linear programming with mixed multiple objectives, Omega, 1977, 5 (2), pp. 193-205.

15. I. M. Stancu-Minasian, Stochastic Programming with Multiple Objective Functions, Ed. Academiei Romane and D. Reidel Publishing Company, Dordrecht, 1984.

16. I. M. Stancu-Minasian and S. Tigan, The minimum-risk approach to special problems of mathematical programing. The distribution function of the optimal value, Rev. Anal. Numér. Théor. Approx., 1984, 13 (2), pp. 175-187.

17. I. M. STANCU-Minasian and S. Tigan, The minimum risk approach to max-min bilinear programming, An. Stiint. Univ. "Al. I. Cuza" Iasi, Sect. I-a Math. 1985, (N. S.), 31 (2), pp. 205-209.

18. I. M. Stancu-Minasian and S. Tigan, The stochastic linear-fractional max-min problem, Itinerant Seminar on Functional Equations, Approximation and Convexity, (Cluj-Napoca, 1987), 275-280, Preprint 87-6, Univ "Babes-Bolyai", Cluj-Napoca, 1987.

19. I. M. Stancu-Minasian and S. Tigan, Criteriul riscului minim in programarea stochastica, Lucrarile Sesiunii Stiintifice a Centrului de Calcul al Universitatii Bucuresti, 20-21 februarie 1987, pp. 392-397.

20. I. M. Stancu-Minasian and S. Tigan, On some fractional programming models occurring in minimum-risk problems, in: Generalized Convexity and Fractional Programming with Economic Applications, A. Cambini, E. Castagnoli, L. Martein, P. Mazzoleni and S. Schaible (eds.), Proceedings of the International Workshop on "Generalized Concavity, Fractional Programming and Economic Applications" held at the University of Pisa, Italy, May 30 - June 1, 1988. Lecture Notes in Economics and Mathematical Systems 345, Springer-Verlag, 1990, pp. 295-324.

21. I. M. StANCU-Minasian and S. Tigan, Generalized pseudofractional max-min problem, Itinerant Seminar on Functional Equations, Approximation and Convexity, (ClujNapoca, 1988), pp. 295-302, Preprint 88-6, Univ. "Babes-Bolyai", Cluj-Napoca, 1988.

22. I. M. Stancu-Minasian and S. Tigan, Multiobjective mathematical programming with inexact data, R. SLowinski and J. TEGHEM (eds.), Stochastic versus Fuzzy Approaches to Multiobjective Mathematical Programming under Uncertainty, Kluwer Academic Publishers, 1990, pp. 395-418. 
23. S. Tigan, On the max-min nonlinear fractional problem, Rev. Anal. Numér. Théor. Approx., 1980, 9 (2), pp. 283-288.

24. S. TIGAN, A parametrical method for max-min nonlinear fractional problems, Itinerant Seminar on Functional Equations, Approximation and Convexity, (Cluj-Napoca, 1983), pp. 175-184, Preprint, 83-2, Univ. "Babes-Bolyai", Cluj-Napoca, 1983.

25. S. Tigan, A quasimonotonic max-min programming problem with linked linear constraints, Itinerant Seminar on Functional Equations, Equations, Approximation and Convexity, (Cluj-Napoca, 1986), pp. 279-284, Preprint 86-7, Univ. "BabesBolyai", Cluj-Napoca, 1986.

26. S. Tigan, Numerical methods for solving some max-min pseudo-fractional prroblems, Proceedings of the Second Symposium of Mathematics and its Applications, Timisoara October 30-31, 1987, Polytechnical institute Timisoara (Romania), 1988, pp. 93-97.

27. S. Tigan, On some procedures for solving fractional max-min problems, Rev. Anal. Numér. Théor. Approx., 1988, 17 (1), pp. 73-91.

28. S. Tigan, On a quasimonotonic max-min problem, Rev. Anal. Numér. Théor. Approx., 1990,19 (4), pp. 85-91.

29. S. Tigan and I. M. Stancu-Minasian, The stochastic max-min problem, Cahiers Centre Études Rech. Opér., 1985, 27 (3-4), pp. 153-158.

30. S. Tigan and I. M. Stancu-Minasian, On a bicriterion max-min fractional problem, Rev. Anal. Numér. Théor. Approx., 1991, 20 (1-2), pp. 117-125.

31. P. WolfE, Determinateness of polyhedral games, in Linear Inequalities and Related Systems, Eds. H. W. KunN, A. W. TuCKER, Princeton University Press, Princeton, 1956. 